\title{
Meta-análisis: Más evidencia a favor de la efectividad del apoyo domiciliario para ancianos
}

Effectiveness of home based support for older people:systematic review and meta-analysis. Elkan R, Kendrick D, Dewey $M$ et al. $B M J$ 2001;323:719-25.

\section{Objetivo}

Investigar en la bibliografía disponible la efectividad de los programas de apoyo domiciliario que ofrecen promoción de la salud y cuidados preventivos para ancianos.

\section{Lugar}

Revisores ingleses a partir de estudios provenientes de los Estados Unidos de Norteamérica, Canadá, Dinamarca, el Reino de los Países Bajos y el Reino Unido.

\section{Diseño}

Meta-análisis*

\section{Fuente y selección de estudios}

Los autores realizaron una revisión sistemática de estudios que evaluaron los efectos de las visitas domiciliarias a personas de 65 y más años.Se incluyeron sólo 15 estudios clínicos prospectivos controlados de los cuales en 13 la selección de los participantes fue aleatoria, y no aleatoria (cuasi-experimental*) en los dos estudios restantes.

\section{Participantes}

En nueve de los trabajos se enrolaron ancianos de la población general mientras que en los restantes seis se incluyeron ancianos "frágiles" que presentaban diferentes tipos de comorbilidades asociadas.

\section{Medición de resultados principales}

En los estudios incluidos se evaluaron puntos finales de prevención como mortalidad, admisiones hospitalarias, admisiones a centros de tercer nivel, capacidad funcional y calidad de vida.

\section{Intervención}

Los ancianos en todos los grupos de intervención recibieron visitas domiciliarias en el marco de diversos programas.

\section{Resultados principales}

De los ocho trabajos que midieron la mortalidad como punto final principal en la población general, se evidenció una reducción significativa de la misma como consecuencia del programa de visitas con un odds ratio $\left(\mathrm{OR}^{*}\right)$ de 0,76 (IC $95 \% 0,64$ a 0,89 ). Cinco estudios demostraron una reducción de la mortalidad entre los ancianos "frágiles" enrolados en programas de visitas domiciliarias con un OR de 0,72 (IC 95\% 0,52 a 0,97). Además, las visitas domiciliarias se asociaron a una menor tasa de admisión a centros de tercer nivel entre los miembros de la población anciana general con un OR de 0,65 (IC 95\% 0,46 a 0,91). El análisis no permitió demostrar reducción alguna de la tasa de admisión hospitalaria, mejoría de la capacidad funcional o de la calidad de vida tanto en la población anciana general como en la frágil.

\section{Conclusiones}

Los programas de visitas domiciliarias de ancianos pueden reducir la mortalidad y la admisión a centros de tercer nivel.

\section{Comentario}

Los programas de visitas o cuidados domiciliarios se han difundido alrededor del mundo durante los últimos 20 años y comprenden una amplia gama de prestaciones médicas que van desde visitias prev entivas a cuidado de pacientes crónicos, pasando por una variedad de intervenciones para el tratamiento de enfermedades agudas.Todos ellos gozan de gran aceptación tanto por parte de los servicios de salud como de sus beneficiarios. No obstante lo previo, los estudios clínicos controlados aleatorizados para evaluar la efectividad de estos programas son escasos y no han enrolado una gran cantidad de participantes. Debido a esto, las revisiones sistemáticas ${ }^{*}$ y meta-análisis* de dichos trabajos resultan de gran importancia.

El presente meta-análisis* hace hincapié en visitas preventivas y de promoción de la salud, y concluye que este tipo de programas de visitas domiciliarias de ancianos reducen la mortalidad y la admisión a centros de cuidados prolongados.Sin embargo, llama la atención que no se hubiera comprobado la reducción en la tasa de admisiones hospitalarias. Una explicación para este hallazgo podría ser que las visitas domiciliarias hubieran sido útiles para identificar ancianos con indicación de internación y que de no haberse producido, se les hubiera negado la posibilidad de una internación a un hospital general. Esta especulación se encuentra avalada en parte por el hecho de que sí se registró una disminución en la mortalidad general.
Otro punto que debe ser enfatizado es la imposibilidad del presente estudio en demostrar una mejoría de la capacidad funcional y la calidad de vida en ancianos bajo programas de visitas domiciliarias. Lamentablemente, en la mayoría los estudios revisados no fueron utilizadas herramientas validadas con la suficiente sensibilidad para detectar cambios sutiles para estos puntos finales en población anciana 1 .Estos puntos finales son más complejos y difíciles de analizar en un meta-análisis*. Existen muchos factores adicionales que dificultan el análisis de la relación entre la intervención específica y la mejoría de ciertos puntos finales. Por un lado, la mayoría de los trabajos proveen sólo información general acerca de la naturaleza de la intervención. Por el otro, la mayoría de los estudios revisados no aportan la información suficiente para determinar el grado de adherencia de los sujetos a las intervenciones. Una adherencia insuficiente podría indicar un inadecuado diseño de algunas intervenciones para satisfacer algunas necesidades específicas de los gerontes estudiados?

En conclusión, si bien el presente meta-análisis* muestra que los programas de visitas domiciliarias para ancianos son útiles para reducir la mortalidad y la admisión a centros de tercer nivel, es necesario diseñar estudios para identificar qué componentes de la intervención son más efectivos y cuáles las poblaciones expuestas a obtener mayores beneficios.

Dr. Pablo Przygoda [ Servicio de Clínica Médica.Hospital Italiano de Buenos Aires ]

Referencias

1.van Rossum E, Frederiks CMA, Philipsen II, et al. Effects of preventive home visits to elderly people.BMJ 1993;307:27-32.

2.van Haastregt JCM, Diederiks JPM, van Rossum E, et al. Effects of preventive home visits to elderly people living in the community:systematic review.BMJ 2000;320:754-8. 